\title{
A simple approach improving the performance of urine reagent strips for rapid diagnosis of urinary schistosomiasis in Nigerian schoolchildren
}

\author{
Uade Samuel Ugbomoiko', Victor Dalumo', Liana Ariza², \\ Fernando Schemelzer Moraes Bezerra ${ }^{3}$, Jorg Heukelbach ${ }^{4,5} /+$
}

\begin{abstract}
'Department of Zoology, University of Ilorin, Nigéria ²Programa de Pós-graduação em Ciências Médicas Departamento de Análises Clínicas ${ }^{4}$ Departamento de Saúde Comunitária, Faculdade de Medicina, Universidade Federal do Ceará, Rua Professor Costa Mendes 1608 5o andar, 60430-140 Fortaleza, Ceará, Brasil ${ }^{5}$ Anton Breinl Centre for Public and Tropical Medicine, School of Public Health, Tropical Medicine and Rehabilitation Sciences, James Cook University, Townsville, Queensland, Australia
\end{abstract}

In Nigeria, schistosomiasis, caused predominantly by the species Schistosoma haematobium, is highly endemic in resource-poor communities. We performed a school-based survey in two rural communities in Osun State (Southwestern Nigeria) and assessed macrohaematuria, microhaematuria and proteinuria as indirect indicators for the presence of disease. Urine samples were inspected macroscopically for haematuria and screened for microhaematuria and proteinuria using urine reagent strips. The microscopic examination of schistosome eggs was used as the gold standard for diagnosis. In total, 447 schoolchildren were included in this study and had a 51\% prevalence of urinary schistosomiasis. The sensitivity of microhaematuria (68\%) and proteinuria (53\%) for infection with $\mathrm{S}$. haematobium was relatively low. In patients with a heavy infection $(\geq 500$ eggs $/ 10 \mathrm{~mL})$, the sensitivity of microhaematuria was high (95\%). When the presence of macrohaematuria and the concomitant presence of microhaematuria and proteinuria were combined, it revealed a sensitivity of $63 \%$, a specificity of $93 \%$ and a positive predictive value of 91\%. Macrohaematuria also showed high specificity (96\%) and a positive predictive value of $92 \%$, while sensitivity was $<50 \%$. These data show that combining urine reagent strip tests (presence of proteinuria and microhaematuria) and information on macrohaematuria increased the accuracy of the rapid diagnosis of urinary schistosomiasis in an endemic rural West African setting. This simple approach can be used to increase the quality of monitoring of schistosomiasis in schoolchildren.

Key words: urinary schistosomiasis - urine reagent strips - prevalence - rapid assessment - Nigeria

There are an estimated 200 million people infected with schistosomiasis throughout the world, and this disease is responsible for the loss of approximately 1.5 million DALYs/year (Chitsulo et al. 2000, Gryseels et al. 2006, Mathers et al. 2007). In sub-Saharan Africa, up to 280,000 annual deaths have been attributed to schistosomiasis (Gryseels et al. 2006). Urinary schistosomiasis remains an intractable parasitic disease, associated with populations living in poverty in subSaharan Africa and it has placed an enormous toll on the health sectors of affected countries (Chitsulo et al. 2000, Gryseels et al. 2006).

In all the geographical zones of Nigeria, there has been an unprecedented increase in disease prevalence in recent years, which is associated with the poor management of water resource schemes (Ofoezie \& Asaolu

Financial support: CNPq/PROÁFRICA (490429/2007-6). LA received a PhD scholarship from CAPES (Brazil).

+Corresponding author: heukelbach@web.de

Received 13 August 2008

Accepted 3 March 2009
1997, Ofoezie 2002, Mafiana et al. 2003). The predominant species in this country is Schistosoma haematobium, which most commonly presents with haematuria, suprapubic pain and pain while urinating (Okoli \& Iwuala 2004).

The Nigerian Schistosomiasis Control Programme has advocated mass administration of praziquantel, particularly in high risk areas, in order to reduce morbidity (WHO 2002). School-aged children are particularly vulnerable to infection and severe disease and are thus the focus of the Control Programme. However, to be effective, control strategies in resource-poor settings require rapid and accurate identification of disease, monitoring of populations at risk, adequate treatment and quantification of infection rates using limited economic and human resources (Mafe et al. 2000, French et al. 2007).

Since urinary schistosomiasis is a common cause of blood in the urine of people living in endemic areas, the presence of haematuria is commonly used as a rapid assessment method for identifying infected individuals and communities at risk.

In the present school-based study, we report the prevalence and intensity of urinary schistosomiasis in two Nigerian rural communities. To improve the rapid assessment of schistosomiasis in this setting, we assessed macrohaematuria, microhaematuria and proteinuria as 
indirect disease markers and described diagnostic performances based on these individual markers and their combinations.

\section{MATERIALS AND METHODS}

A survey was conducted in the four schools of the Ilobu and Erin-Osun communities, which consist of two contiguous villages with similar socio-economic and cultural characteristics in the Osun State of Southwestern Nigeria. The detailed characteristics of these communities have previously been described (Ugbomoiko et al. 2009, unpublished observations). Briefly, both communities border the Erinle Dam, which was constructed in the 1990s and is used principally for flood control, fishing and as a water supply. The communities are provided with primary and secondary schools, health centres and functional boreholes. The approximately 5,000 inhabitants of both communities are predominantly subsistence farmers and petty traders of the Yoruba ethnic group.

All schoolchildren, from both primary and secondary schools, were eligible for this study. After giving consent, the participants received pre-labelled screw-capped plastic containers for urine collection. To avoid false positive results, girls of child-bearing age who had menstruated within five days before sample collection were excluded from analysis. The freshly passed mid-day urine samples (collected between 10-14 h) were inspected macroscopically for gross haematuria and then screened for microhaematuria and proteinuria using commercially available urine reagent strips (medi-Test Combi 9, Analyticon Biotechnologies, Germany). The strip testing was performed in accordance with the manufacturer's instructions. The specimens were then transported to the laboratory within $4 \mathrm{~h}$ of collection and processed for microscopic examination of schistosome eggs.

Processing and egg counting followed the syringe filtration technique, which we considered the gold standard for diagnosis of schistosomiasis (Guyatt et al. 1999, WHO 1983, Lengeler et al. 1993). Two subsamples of 10 $\mathrm{mL}$ were drawn into plastic syringes from each wellmixed sample and strained through a nylon filter. The filter was then examined under the microscope, and $S$. haematobium eggs were counted.

Data were entered into an Excel spreadsheet, checked for entry errors and transferred into SPSS for Windows (version 11.0, SPSS Inc, Chicago, USA) for analysis. The sensitivity (number of individuals with a positive rapid test/individuals with a positive reference test), specificity (number of individuals with a negative rapid test/individuals with a negative reference test), the negative predictive value (probability that an individual with a negative rapid test is, in fact, free from disease) and the positive predictive value (probability that an individual with a positive rapid test is, in fact, diseased) were calculated for different combinations of rapid tests, as compared to the gold standard. Chi-square and ANOVA tests were applied to compare relative frequencies and the arithmetic means of egg counts between groups, respectively.

Ethics - At the time of this study, a formal ethical committee in state of Osun did not exist. Permission to perform this study was obtained from the local governments, community leaders and school heads. Informed consent was obtained from the study participants and their parents after a detailed explanation of the objectives of the study. All infected schoolchildren were referred to medical personal in the Area Office for praziquantel treatment.

\section{RESULTS}

In total, 447 schoolchildren and pre-schoolchildren were included in this study. Among the participants, $248(56 \%)$ were male and the median age was 11 years (interquartile range: 9-13 years; range: 3-17 years). Prevalence and intensity of urinary schistosomiasis are depicted in Table I. The prevalence was significantly higher in Ilobu village. In boys, prevalence of infection was significantly higher than in girls and the mean egg count was approximately twice as high; however, the difference was not statistically significant (Table I). The prevalence increased significantly with age and showed a peak in participants who were $\geq 15$ year-olds, with $78 \%$ of individuals infected.

Table II depicts the sensitivity, stratified by intensity of infection, of macrohaematuria, microhaematuria, proteinuria and their combinations in the diagnosis of urinary schistosomiasis. In general, sensitivities were low, but increased with severity of infection. In almost all patients presenting with heavy infection (95\%), microhaematuria was observed. In contrast, macrohaematuria was detected in only $45 \%$ of the total of patients with schistosomiasis (Table II). Considering the rapid assessment as positive in the presence of macrohaematuria or, alternatively, in the presence of concomitant microhaematuria and proteinuria, an overall sensitivity of $63 \%$ and of $90 \%$ in heavy infections was revealed.

In Table III, the diagnostic performance values of the indicators macrohaematuria, microhaematuria, proteinuria and their different combinations are given using the presence of eggs in urine as the gold standard for diagnosis. The highest overall sensitivity was found for microhaematuria (68\%), with a specificity of $83 \%$. However, the specificity was highest $(96 \%)$ when the concomitant presence of microhaematuria and proteinuria was used as an infection marker. Macrohaematuria also showed a very high specificity and positive predictive value $(96 \%$ and $92 \%$, respectively), but sensitivity was less than $50 \%$ (Table III). To combine the benefits of all diagnostic approaches, we assessed the accuracy of the concomitant presence of macrohaematuria or the presence of microhaematuria and proteinuria. This improved sensitivity to $63 \%$ while maintaining a high specificity of $93 \%$ (Table III) and it indicates that there were $37 \%$ false negative and $7 \%$ false positive results. In contrast, using a rapid test based merely on the presence of microhaematuria and proteinuria, or on the presence of macrohaematuria, resulted in a proportion of false negative results as high as $62 \%$ and $55 \%$, respectively.

\section{DISCUSSION}

Our data show that combining indirect disease marker information obtained from both urine reagent strip 
tests and presence of macrohaematuria increased the accuracy of rapid diagnosis of urinary schistosomiasis in schoolchildren of two highly endemic rural communities in Nigeria.

When the macrohaematuria information was combined with data from urine reagent strips, a high positive predictive value was maintained with the benefit of an increased negative predictive value (the probability of children with a negative test being disease-free). Thus, this approach can be considered a cost-effective measure for increasing the quality of data on schistosomiasis infection in schoolchildren located in highly endemic communities.

With this approach, the positive predictive value of 91\% (the probability of schistosomiasis among children with a positive test) was high and similar to the positive predictive value of macrohaematuria (92\%). This indicates that almost all schoolchildren with positive rapid tests were, in fact, infected. However, since the predictive values depend on the prevalence of disease and intensity of infection, in low endemic settings the positive predictive value may be different. The negative predictive value of $63 \%$ indicates that the probability of schoolchildren having the disease, in the absence of macrohaematuria, is $37 \%$. This probability was reduced to $29 \%$ when using our combined approach, but it is still high and should be taken into account when planning mass drug administration and monitoring intervention measures.

Previously, other authors have combined data on haematuria and proteinuria, improving the diagnostic performance of disease markers (Feldmeier et al. 1982, Mott et al. 1983). However, these authors did not integrate information gathered from reagent strips and on macrohaematuria. Our data further confirm previous studies on the diagnostic performance of microhaema- turia and macrohaematuria, when compared to urine egg count as the gold standard (Mott et al. 1983, Eltoum et al. 1992, Lengeler et al. 1993, Mafe 1997, Guyatt et al. 1999). Haematuria and proteinuria have been widely used as indicators for urinary schistosomiasis since the 1980s and usually microhaematuria is considered more sensitive and specific than proteinuria (Mott et al. 1983, 1985, Murare \& Taylor 1987, Taylor et al. 1990, Eltoum et al. 1992). Similar to our data, Mafe (1997) described sensitivity $(69 \%)$ and specificity $(80 \%)$ of microhaematuria for the diagnosis of schistosomiasis in Nigeria, albeit in the general population. In that study, the diagnostic performance of macrohaematuria was comparable to our results (Mafe 1997). French et al. (2007) observed, in a longitudinal study of schoolchildren from Zanzibar, consistently higher sensitivity $(\geq 77 \%)$ and specificity $(\geq$ 97\%) of microhaematuria. Besides regional differences, the increased accuracy in this study may also be explained by the varying quality of reagent strips from different producers. Interestingly, the diagnostic values from the Zanzibar study remained relatively stable during various assessments, even when the prevalence was reduced during a control programme (French et al. 2007).

The presence of visible blood in urine (macrohaematuria) in settings without the availability of urine reagent strips is used as a specific rapid diagnostic marker for $S$. haematobium infection (Red Urine Study Group 1995, Mafe 1997, Ofoezie et al. 1997, Mafe et al. 2000, Anosike et al. 2001, Lengeler et al. 2002). Therefore, the almost $100 \%$ specificity found in our study was not a surprise. However, most of those studies were performed on a limited number of schoolchildren, and the accuracy of the results was influenced by prevalence, age and sex (Mott et al. 1983, Ansell et al. 1997, Guyatt et al. 1999).

TABLE I

Prevalence and intensity of infection of urinary schistosomiasis in schoolchildren of two endemic communities in Nigeria $(\mathrm{n}=447)$

\begin{tabular}{|c|c|c|c|}
\hline & $\begin{array}{c}\text { Positive/total } \\
\mathrm{n}\end{array}$ & $\begin{array}{c}\text { Prevalence } \\
(95 \% \text { confidence interval) } \%\end{array}$ & $\begin{array}{c}\text { Mean }( \pm \mathrm{SD}) \\
\text { egg count } / 10 \mathrm{~mL} \text { urine }\end{array}$ \\
\hline \multicolumn{4}{|l|}{ Villages } \\
\hline Erin-Osun & $114 / 246$ & $46.3(39.9-52.8)$ & $107.05 \pm 476.09$ \\
\hline Ilobu & $113 / 201$ & $56.2(49.1-63.2)$ & $138.78 \pm 336.35$ \\
\hline p-value & & 0.038 & 0.426 \\
\hline \multicolumn{4}{|l|}{ Sex } \\
\hline Male & $141 / 248$ & $56.9(50.4-63.1)$ & $155.06 \pm 503.38$ \\
\hline Female & $86 / 199$ & $43.2(36.2-50.4)$ & $76.26 \pm 275.69$ \\
\hline p-value & & 0.004 & 0.057 \\
\hline \multicolumn{4}{|c|}{ Age group (years) } \\
\hline $1-4$ & $4 / 11$ & $36.4(10.9-69.2)$ & $2.27 \pm 4.78$ \\
\hline $5-9$ & $45 / 137$ & $32.8(25.1-41.4)$ & $53.74 \pm 206.69$ \\
\hline $10-14$ & $147 / 259$ & $56.8(50.5-62.9)$ & $142.25 \pm 487.16$ \\
\hline $15-17$ & $31 / 40$ & $77.5(61.5-89.2)$ & $249.98 \pm 498.05$ \\
\hline p-value & & $<0.001$ & 0.030 \\
\hline Total & $227 / 447$ & $50.8(46.0-55.5)$ & $121.32 \pm 418.92$ \\
\hline
\end{tabular}



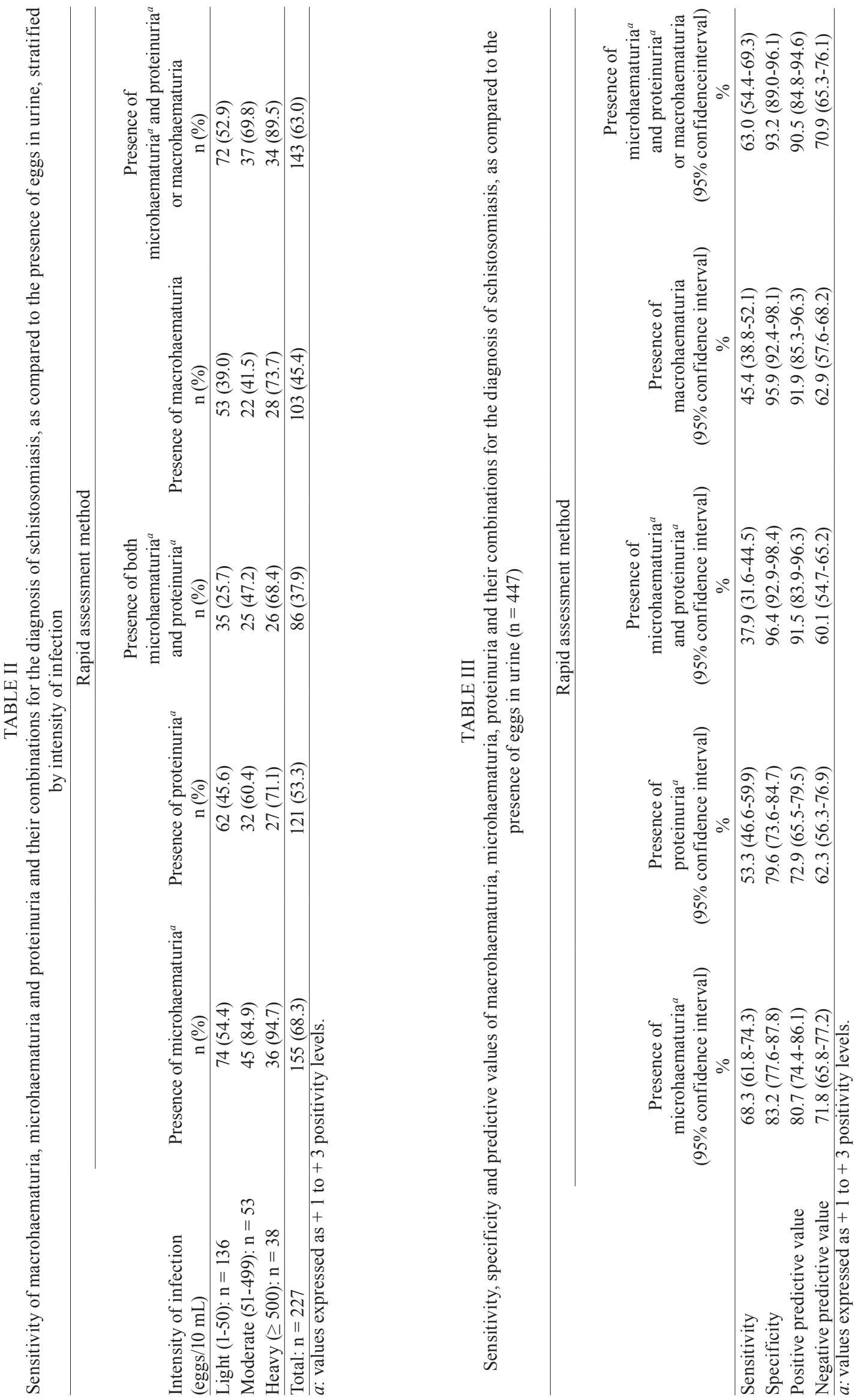
In addition, the sensitivity of macrohaematuria is usually low, but may increase in the presence of heavy infection (Taylor et al. 1990, Mafe 1997), as also shown in our study. Haematuria can be seen as a sign of tissue damage occurring principally in severe infections. Thus, in extremely resource-poor settings, where no urine reagent strips are available, the mere presence of macrohaematuria may be used as an indicator for infection and to detect the most severely infected individuals.

Macrohaematuria may also be assessed using questionnaires, which generally have an acceptable diagnostic performance (Lengeler et al. 2002). However, the specificity and/or sensitivity of questionnaire-based approaches vary among studies and socio-cultural settings and the accuracy of this approach was low in several cases (Guyatt et al. 1999, Poggensee et al. 2000, Lengeler et al. 2002, Stothard et al. 2002).

The present study further revealed a high rate of $S$. haematobium transmission in the rural population around the Erinle Dam in Southwestern Nigeria. The overall prevalence of schistosomiasis in schoolchildren (51\%) was about four times higher than the previously estimated prevalence in Nigeria's general population (13\%) (Oladejo \& Ofoezie 2006). A recently conducted study in two rural communities in the same area reported a similar prevalence of $47 \%$ (Oladejo \& Ofoezie 2006), indicating that infection has remained unabated in this and, most likely, other dam regions of Nigeria. Age and sex-related prevalence and the intensity of infection in the present study were similar to those reported from other West African endemic foci (Okoli \& Odaibo 1999, Mafe et al. 2000, Okoli \& Iwuala 2004, Oladejo \& Ofoezie 2006).

Our study is subject to several limitations. First, the survey only included schoolchildren and as such, data cannot be inferred to the general population. It is known that the diagnostic performance of haematuria and proteinuria decreases with increasing age, but it is usually stable in children and teenagers (Mott et al. 1983, Mafe 1997). However, combining reagent strips with the macrohaematuria observations will probably increase the diagnostic accuracy when applied to the general population. Secondly, the results may only be valid in settings with a high prevalence of schistosomiasis. In areas with a lower prevalence and where infection intensity is consequently lower, diagnostic performance may be less reliable (Hammad et al. 1997). However, another study has shown that rapid assessment using reagent strips may not vary considerably, even when prevalence is decreased (French et al. 2007). An additional shortcoming of our study is its cross-sectional nature: we did not assess day-to-day variation of egg excretion or haematuria, which may have influenced diagnostic performance. In fact, the accuracy of macrohaematuria, as an indicator for infection, may be better when considering day-today variations (Savioli et al. 1990). When the assessment of macrohaematuria is repeated several times during a period of a few days, the accuracy of this indicator is expected to increase.

It can be concluded that new strategies are needed to improve the control and monitoring of urinary schistosomiasis in Nigerian dam areas and in other countries with highly endemic areas. These approaches should be validated by performing studies in other African schools in the endemic areas. If urine reagent strips are available, a combined approach of directly observing macrohaematuria and the concomitant presence of proteinuria and microhaematuria may improve the performance of rapid diagnosis. In resource-poor populations, the proposed approach is feasible since no additional costs and minimal logistic efforts are needed when macrohaematuria data are simply added to the analysis of microhaematuria and proteinuria, as determined by urine reagent strips.

\section{REFERENCES}

Anosike JC, Nwoke BEB, Njoku AJ 2001. The validity of haematuria in the community diagnosis of urinary schistosomiasis infection. $J$ Helminth 75: 223-225.

Ansell J, Guyatt HL, Hall A, Kihamia C, Kivogo J, Ntimbwa P, Bundy D 1997. The reliability of self reported blood in urine and schistosomiasis as indicators of Schistosoma haematobium in schoolchildren: a study in Muheza district, Tanzania. Trop Med Int Health 2: $1180-1189$.

Chitsulo L, Engels D, Montresor A, Salvioli L 2000. The global status of schistosomiasis and its control. Acta Trop 77: 41-51.

Eltoum IA, Sulaiman S, Ismail BM, Ali MMM, Elfatih M, Homeida MMA 1992. Evaluation of haematuria as an indirect screening test for schistosomiasis haematobium: a population-based study in the white Nile Province, Sudan. Acta Trop 51: 151-157.

Feldmeier H, Doehring E, Daffalla AA 1982. Simultaneous use of a sensitive filtration technique and reagent strips in urinary schistosomiasis. Trans R Soc Trop Med Hyg 76: 416-442.

French MD, Rollinson D, Basáñez MG, Mgeni AF, Khami IS, Stothard JR 2007. School-based control of urinary schistosomiasis on Zanzibar, Tanzania: monitoring micro-haematuria with reagent strips as a rapid urological assessment. $J$ Ped Urol 3: 364-368.

Gryseels B, Polman K, Clerinx J, Kestens L 2006. Human schistosomiasis. Lancet 368: 1106-1118.

Guyatt H, Brooker S, Lwambo NJS, Siza JE, Bundy DAP 1999. The performance of school-based questionnaires of reported blood in urine in diagnosing Schistosoma haematobium infection: patterns by age and sex. Trop Med Int Health 4: 751-757.

Hammad TA, Gabr NS, Talaat MM, Orieby A, Shawky E, Strickland GT 1997. Haematuria and proteinuria as predictors of Schistosoma haematobium infection. Am J Trop Med Hyg 57: 363-367.

Lengeler C, Mshinda H, Morona D, deSavigny D 1993. Urinary schistosomiasis: testing with urine filtration and reagent sticks for haematuria provides a comparable estimate Acta Trop 53: 39-50.

Lengeler C, Utzinger J, Tanner M 2002. Questionnaires for rapid screening of schistosomiasis in sub-Saharan Africa. Bull World Health Organ 80: 235-242.

Mafe MA 1997. The diagnostic potential of three indirect tests for urinary schistosomiasis in Nigeria. Acta Tropica 68: 277-284.

Mafe MA, von Stamm T, Utzinger J, Goran EK, Goran N 2000. Control of urinary schistosomiasis: an investigation into the effective use of questionnaires to identify high-risk communities and individuals in Niger State, Nigeria. Trop Med Int Health 5: 53-63.

Mafiana CF, Ekpo UF, Ojo DA 2003. Urinary schistosomiasis in preschool children in settlement around Oyan reservoir in Ogun State, Nigeria: implication for control. Trop Med Int Health 8: 78-82. 
Mathers CD, Ezzati M, Lopez AD 2007. Measuring the burden of neglected tropical diseases: the global burden of disease framework. PloS Negl Trop Dis 1: e114.

Mott KE, Dixon H, Osei-Tutu E, England EC 1983. Relation between intensity of Schistosoma haematobium infection and clinical haematobium and proteinuria Lancet 321: 1005-1008.

Mott KE, Dixon H, Osei-Tutu E, England EC, Ekue K, Tekle A 1985. Evaluation of the reagent strips in urine tests for detection of Schistosoma haematobium infection: a comparative study in Ghana and Zambia Bull World Health Organ 63: 125-133.

Murare HM, Taylor P 1987 Haematuria and proteinuria during Schistosomiasis haematobium infection: relationship to intensity of infection and the value of chemical reagent strips for pre and posttreatment diagnosis. Trans R Soc Trop Med Hyg 81: 426-430.

Ofoezie IE 2002. Human health and sustainable water resour development in Nigeria: schistosomiasis in artificial lakes. Nat Resour Forum 26: 150-160.

Ofoezie IE, Asaolu SO 1997. Water level regulation and the control of schistosomiasis transmission: a case in Oyan Reservoir, Ogun State, Nigeria. Bull World Health Organ 75: 436-441.

Ofoezie IE, Asaolu SO, Christensen NO, Madsen H 1997. Pattern of infection of Schistosoma haematobium in lakeside resettlement communities at the Oyan Reservoir in Ogun State, Southwest Nigeria. Ann Trop Med Parasitol 91: 187-197.

Okoli CG, Iwuala MOE 2004. The prevalence, intensity and clinical signs of urinary schistosomiasis in Imo State, Nigeria. $J$ Helminthol 78: $337-342$.

Okoli EI, Odaibo AB 1999. Urinary schistosomiasis among schoolchildren in Ibadan, an urban community in Southwestern Nigeria. Trop Med Int Health 4: 308-315.

Oladejo SO, Ofoezie IE 2006. Unabated schistosomiasis transmission in Erinle River Dam, Osun State, Nigeria: evidence of neglect of environmental effects of development projects. Trop Med Int Health 11: 843-850.

Poggensee G, Krantz I, Kiwelu I, Feldmeier H 2000. Screening on Tanzanian women of childbearing age for urinary schistosomiasis: validity of urine reagent strip readings and self-reported symptoms. Bull World Health Organ 78: 542-548.

Red Urine Study Group 1995. Identification of high risk communities for schistosomiasis in Africa: a multi-country study, Social and Economic Research Project Reports 15, World Health Organization, Geneva, $52 \mathrm{pp}$.

Savioli L, Hatz C, Dixon H, Kisumku UM, Mott KE 1990. Control of morbidity due to Schistosoma haematobium on Pemba Island: egg excretion and haematuria as indicators of infection. Am J Trop Med Hyg 43: 289-295.

Stothard JR, Mgeni AF, Khamis S, Seto E, Ramsan M, Rollinson D 2002. Urinary schistosomiasis in schoolchildren on Zanzibar Island (Unguja), Tanzania: a parasitological survey supplemented with questionnaires. Trans R Soc Trop Med Hyg 96: 507-514.

Taylor P, Chandiwana SK, Matanhire D 1990. Evaluation of the reagent strip test for haematuria in the control of Schistosoma haematobium infection in schoolchildren. Acta Trop 47: 91-100.

Ugbomoiko US, Dalumo V, Obiezue RNN, Ofoezie IE 2009. Socio-environmental factors in ascariasis infection among school aged children in Ilobu Osun State, Nigeria. Trans $R$ Soc Trop Med Hyg, 103: 223-228.

WHO - World Health Organization 1983. Diagnostic techniques in schistosomiasis control. Unpublished document (WHO/SCHIS$\mathrm{TO} / 83.69)$.

WHO - World Health Organization 2002. Prevention and control of schistosomiasis and soil-transmitted helminthiasis, Technical Report Series 912, World Health Organization, Geneva, 63 pp. 